\title{
EVALUACIÓN Y MONITOREO MICROBIOLÓGICO DE FERTILIDAD DE HUEVOS DE AVESTRUCES MEDIANTE INCUBACIÓN ARTIFICIAL*
}

\author{
Arauco Villar, Fernando ${ }^{1}$, Pérez Castro, Eleazar² y Mayorga Sánchez, Noemí3 \\ Facultad de Zootecnia de la Universidad Nacional del Centro del Perú
}

\begin{abstract}
RESUMEN
La presente investigación pretende demostrar que el lote de avestruces reproductores de la EEA El MantaroUNCP puede producir huevos fértiles y obtener adecuados índices de incubabilidad y eclosión bajo incubación artificial a 3200 msnm y contribuir al conocimiento de los factores que inciden sobre la fertilidad e incubabilidad en avestruces. El proceso de incubación fue llevado a cabo en la localidad de YanamucloJauja, y los exámenes microbiológicos fueron realizados en el laboratorio de microbiología de la Facultad de Zootecnia - UNCP. El período de investigación comprendió desde abril del 2008 hasta marzo de 2009. Los resultados fueron: el número de huevos producidos para incubación fue de 64 en total para los dos meses de ciclo reproductivo; el promedio del peso de los huevos fue $1,512 \mathrm{Kg}$.; se realizaron 13 cargas de huevos a la incubadora, con un promedio de 4,92 huevos/carga, el peso promedio de los huevos/cargada fue 1,519. Las hembras $N^{0} 1489$ y 1405 fueron las mejores ponedoras con 19 y 14 huevos respectivamente y tuvieron el mejor comportamiento en lo referente a su fertilidad, pues a la primera y segunda ovoscopía presentaron el mayor número de huevos en proceso de incubación. Los mejores machos fueron los № 2042, 1412 y 1331. El peso promedio de los huevos a la $1^{\text {a }}$ ovoscopía fue 1,453 $\mathrm{Kg}$., descartándose 32 huevos y quedando 32 ; y a la $2^{\mathrm{a}}$ ovoscopía fue $1,349 \mathrm{Kg}$, descartándose 16 huevos y quedando 16 . La pérdida de peso de los huevos de la $1^{\mathrm{a}}$ a la $2^{\mathrm{a}}$ ovoscopía en promedio fue $0,124 \mathrm{Kg}$. Hay un nivel de correlación significativo entre los valores de pérdida de peso de los huevos desde el inicio de la incubación hasta la $1^{a}$ ovoscopía, y la $1^{\mathrm{a}}$ y $2^{\mathrm{a}}$ ovoscopía. El \% de fertilidad fue del $50 \%$ y el porcentaje de incubabilidad fue 0 . Los reportes del laboratorio indican presencia de Escherichia coli y Bacillus $s p$, de manera individual o combinada en todas las muestras tomadas, al inicio y al final del ciclo reproductivo de las hembras.
\end{abstract}

Palabras clave: avestruz, incubabilidad, fertilidad, ovoscopía, microorganismos

\footnotetext{
* Este trabajo de investigación fue recibido el 20/03/2009 retornado para su revisión 26/10/2009 y aprobado para su publicación 16/11/2009

1Email: faraucov@hotmail.com

2Email: eleazarperu@yahoo.com

${ }^{3}$ Email: nmayorga@hotmail.com
} 


\begin{abstract}
The present investigation pretends to show that the ostriches lot of the EEA El Mantaro-UNCP can produce fertile eggs and obtain appropriate hatchability and hatching under artificial incubation at 3200 mosl and to contribute to the knowledge of the factors that impact on the fertility and hatchability in ostriches. The incubation process was carried out in the town of Yanamuclo-Jauja, and the microbiológical test was carried out in the Microbiological Laboratory of Zootecnic Faculty - UNCP. The period of investigation was from April 2008 to March 2009. The results were: $N^{0}$ of eggs taken place for incubation was 64 total for the two months of reproductive cycle; the average weight of the eggs was $1,512 \mathrm{Kg}$.; there were carried out 13 loads of eggs to the incubator, with an average of 4,92 eggs/load, the weight average of the eggs/load was 1,519 . The female $N^{0}$ s 1489 and 1405 was the best egg-laying with 19 and 14 eggs respectively and they had the best behavior regarding its fertility, because the first and second candling they presented the biggest number of eggs in incubation process. The best males were the $\mathrm{N}^{0} \mathrm{~s} 2042,1412$ and 1331 . The weight average of the eggs to the $1^{\mathrm{a}}$ candling was $1,453 \mathrm{Kg}$., being discarded 32 eggs and being 32 ; and to the $2^{\mathrm{a}}$ ovoscopy it was $1,349 \mathrm{Kg}$, being discarded 16 eggs and being 16. The loss of weight of the eggs of $1^{\mathrm{a}}$ to the $2^{\mathrm{a}}$ ovoscopy on the average was $0,124 \mathrm{Kg}$. There is a significant correlation level among the values of loss of weight of the eggs from the beginning of the incubation until the $1^{\mathrm{a}}$ ovoscopy and the $1^{\mathrm{a}}$ and $2^{\mathrm{a}}$ ovoscopy. The fertility percentage was $50 \%$ and the hatchability percentage was 0 . The laboratory reports indicate the presence of Escherichia coli and Bacillus sp, in an individual or combined way in all the taken samples, at the beginning and at the end of female reproductive cycle.
\end{abstract}

Key words: ostrich, incubability, fertility, ovoscopy, microorganisms 


\section{INTRODUCCIÓN}

La UNCP a través del Programa de Avestruces de la EEA El Mantaro, se desarrolló un Proyecto de crianza de avestruces para reproducción, con la finalidad de poner a disposición de los potenciales productores en este nuevo rubro de la Estrutiocultura, de huevos embrionados, polluelos y avestruces juveniles, para lo cual se cuenta con un lote de 19 reproductores entre (10 machos y 9 hembras). $\mathrm{Si}$ bien es cierto que el Proyecto se desarrolla desde hace unos 5 años, no se han seguido las pautas técnicas de manejo y alimentación recomendados para esta especie, por diferentes razones. Existe interés en replantear las condiciones de crianza para poder cumplir con el objetivo trazado en el proyecto primigenio, cual es, servir de centro de reproducción de avestruces para los futuros productores de la región y del país. Esta es la motivación principal para ejecutar el presente proyecto de investigación, aportando soluciones al problema de la todavía incierta capacidad reproductiva de machos y hembras y a determinar la factibilidad de incubar artificialmente los huevos en condiciones de altura. El problema planteado fue: ison los avestruces del Programa de Avestruces de la EEA El Mantaro de la UNCP capaces de producir huevos fértiles aptos para la incubación artificial y se puede llevar a cabo este proceso en condiciones de altura (3 $200 \mathrm{msnm}$ )?, y la hipótesis formulada fue que el lote de avestruces puede producir huevos fértiles y obtener adecuados índices de incubabilidad y eclosión bajo incubación artificial a $3200 \mathrm{msnm}$.

\section{MATERIAL Y MÉTODOS}

El período de investigación comprendió desde abril del 2008 hasta marzo del 2009. Se trabajó con 19 avestruces, el total de animales con que cuenta el Programa de Avestruces, de los cuales 9 son hembras y 10 son machos, con una edad de 5 años y medio.

Material de manejo de avestruces (manga de tela, mangos de manejo), corrales para tríos de avestruces, bolsas de plástico estériles, incubadora para huevos de avestruces marca Moldum, nacedora para avestruces marca Moldum, ovoscopio con luz incorporada, estetoscopio, desinfectante, guardapolvos, mascarillas, botas y gorras de tela, cámara fotográfica digital, balanza de precisión digital de mesa, torundas estériles; medios de trasporte, de cultivo (agar nutritivo y agar MacConkey), reactivos para pruebas bioquímicas, microscopio compuesto, estufa, refrigerador y autoclave, mecheros de Bunsen, soporte universal, asa y aguja de Kolle, material de vidrio diverso, equipo de cómputo, material de escritorio.

Metodología para la toma de información: se utilizó el método de investigación de observación directa mediante la técnica de ovoscopia, desde que los huevos son puestos y durante el proceso de la incubación artificial de los mismos, así como el monitoreo microbiológico de muestras cloacales extraídas con torundas estériles directamente desde el urodeum de las hembras en postura.

Los huevos colectados se cargaron a la incubadora luego de pasar por el ovoscopio para determinar la posición de la cámara de aire la misma que quedara en la parte superior, ya que los huevos se colocan en forma vertical, para iniciar el proceso, hasta los 14 días, donde se realizó la primera ovoscopía 0 miraje para observar el desarrollo del embrión descantándose aquellos que no presentaron estas características, luego se realizó la segunda ovoscopía o miraje a los 28 días y se descartó nuevamente aquellos huevos que presentaron muerte embrionaria 0 no presentaron desarrollo embrionario, los huevos aptos se esperó hasta su eclosión a los 42 días. Se procedió a tomar muestras de la cloaca (urodeum) de las hembras al inicio y fin de la investigación con un hisopo estéril y en un medio con suero fisiológico, refrigerándose a $4^{\circ} \mathrm{C}$ hasta el análisis microbiológico.

Variables de evaluación: \% fertilidad, \% incubabilidad y presencia microorganismos en muestras cloacales de hembras.

Diseño experimental: Se trabajó con comparaciones estadísticas considerando las diferentes variables en estudio, empleando estadística inferencial (chi cuadrado, correlación y regresión) usando el paquete estadístico SPSS v.15, considerando los resultados de postura e incubabilidad. 


\section{RESULTADOS}

El número de huevos producidos por las 9 hembras durante la fase de investigación experimental fue 64, relativamente bajo, con un promedio de 7,11 huevos por hembra para los dos meses de ciclo reproductivo.

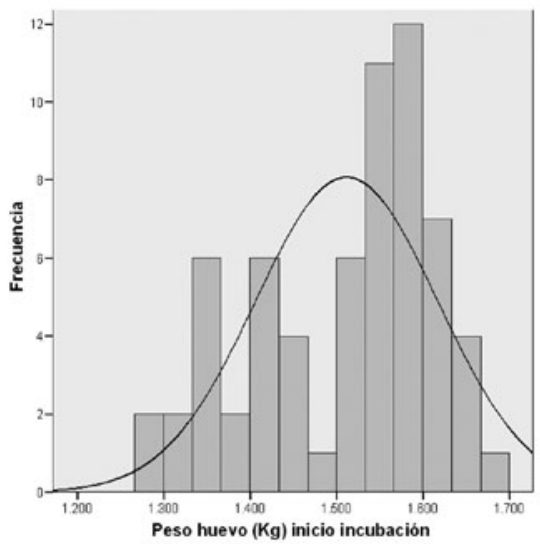

Gráfico 1. Histograma de frecuencias de peso inicial $(\mathrm{Kg})$ de huevos cargados

Promedio peso huevos: $1,512 \mathrm{Kg}$.

Valor máximo: 1,687 Kg. Valor mínimo: 1,285 Kg.

Desviación Standard: 0,105 Variancia: 0,011

Tabla 1. Relación de hembras/huevos incubados

\begin{tabular}{ccccc}
\hline $\begin{array}{c}\boldsymbol{N}^{\mathbf{o}} \\
\text { Hembra }\end{array}$ & Nombre & $\begin{array}{c}\text { Inicio de } \\
\text { postura }\end{array}$ & $\begin{array}{c}\mathbf{1}^{\mathbf{a}} \\
\text { Ovoscopía }\end{array}$ & $\begin{array}{c}\mathbf{2}^{\mathbf{a}} \\
\text { Ovoscopía }\end{array}$ \\
\hline 1336 & Coneja & 07 & 03 & 00 \\
1374 & Pavita & 02 & 01 & 01 \\
1383 & Manchitas & 09 & 01 & 01 \\
1394 & Rizitos & 04 & 00 & 00 \\
1405 & Pata hueca & 14 & 10 & 05 \\
1442 & Alas caídas & 01 & 01 & 01 \\
1446 & Tola & 07 & 00 & 00 \\
1480 & Cuello herido & 01 & 00 & 00 \\
1489 & Cabeza de pato & 19 & 16 & 08 \\
T0TAL & & $\mathbf{6 4}$ & $\mathbf{3 2}$ & $\mathbf{1 6}$ \\
\hline
\end{tabular}

Se realizaron 13 cargas de huevos a la incubadora, con un promedio de 4,92 huevos/carga (rango 1 - 12), haciendo un total de 64 huevos cargados. El peso promedio de los huevos/cargada fue de 1,519, con un rango de 1,403 a 1,610 Kg. La literatura nos manifiesta que normalmente el peso del huevo oscila entre 1,300 y l, $800 \mathrm{Kg}$ de peso, pero pueden haber huevos de hasta $2 \mathrm{~kg}$, y huevos de $900 \mathrm{~g}$. analizando el peso individual de todos los huevos incubados, estos presentaron un promedio de $1,512 \mathrm{Kg}$, con un valor máximo de 1,687 Kg. y un valor mínimo de $1,285 \mathrm{Kg}$. 
Solo 5 hembras produjeron huevos que llegaron hasta los 42 días programados para la incubación. El resto ha producido huevos infértiles 0 se ha producido mortalidad embrionaria precoz 0 a término medio (hasta los 28 días de incubación). Se ha trabajado con 64 huevos para la incubación, y a los 14 días (primera ovoscopía) el $50 \%$ (32 huevos) de ellos fue retirado de la incubación, ya sea por ser infértil o presentar una mortalidad embrionaria precoz (donde suele producirse una autodigestión de un embrión extremadamente pequeño). Dos hembras tuvieron un buen comportamiento en lo referente a su fertilidad ( ${ }^{0} \mathrm{~s} 1489$ y 1405), pues a la primera y segunda ovoscopía presentaron el mayor número de huevos en proceso de incubación. 14 huevos fueron considerados infértiles, pues a la primera ovoscopía no mostraron signos de presencia de desarrollo embrionario, y 18 huevos también fueron retirados luego de este primer miraje por no presentar desarrollo embrionario notorio, compatible con mortalidad embrionaria temprana 0 autodigestión del embrión.

Tabla 2. Tabla de contingencia código de madre * estado del huevo

\begin{tabular}{ccccccc}
\hline Estado del huevo & Infértil & $\begin{array}{c}\text { Mortalidad } \\
\text { embrionaria } \\
\text { temprana }\end{array}$ & $\begin{array}{c}\text { Mortalidad } \\
\text { embrionaria } \\
\text { intermedia }\end{array}$ & $\begin{array}{c}\text { Mortalidad del } \\
\text { polluelo in ovo }\end{array}$ & Total \\
\hline $\begin{array}{c}\text { Código } \\
\text { de la }\end{array}$ & 1336 & 3 & 1 & 3 & 0 & 7 \\
madre & 1374 & 0 & 1 & 0 & 1 & 2 \\
& 1383 & 8 & 0 & 0 & 1 & 9 \\
\hline
\end{tabular}

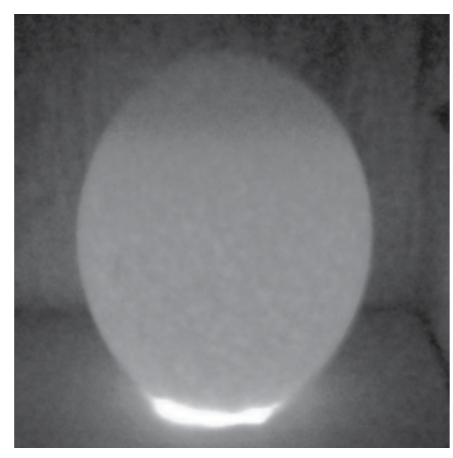

Figura 1. Huevo infértil

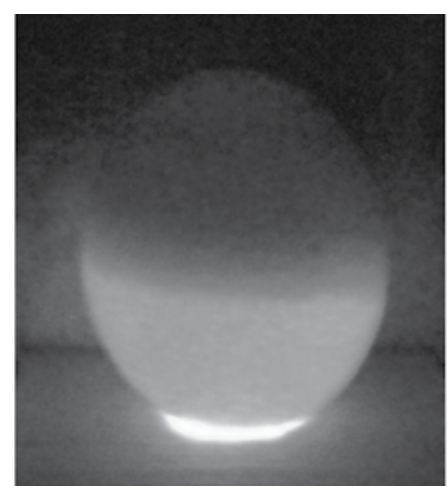

Figura 1. Huevo fértil

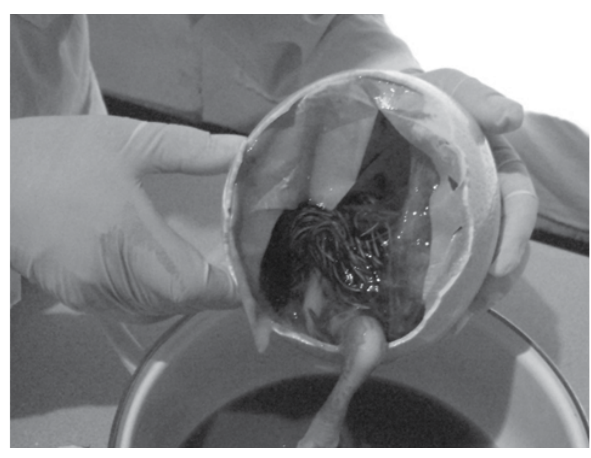

Figura 3. Mortalidad embrionaria tardía

Los machos № 1339, 1355, 1457, 1483; aparentemente no han logrado fecundar a las hembras con las que estuvieron, y hay que tener en cuenta que los espermatozoides de las aves, es su viabilidad y fertilidad por varios días. En el caso de los avestruces ésta llega a los 7 días, de manera que se pueden lograr huevos fértiles durante toda una semana con solo una cópula. Los demás machos han mostrado un comportamiento reproductivo entre bajo a regular, siendo los mejores machos en este rubro los № 2042, 1412 y 1331.

La primera ovoscopía se realizó a los 14 días de iniciado el proceso de incubación. El peso promedio de los huevos fue $1,453 \mathrm{Kg}$, lo que se explica por la pérdida 
de peso de los huevos por el desarrollo embrionario y la deshidratación correspondiente. Luego de esta $1^{\text {a }}$ ovoscopía se descartaron 32 huevos, quedando un igual número para que continúen el proceso de incubación. La pérdida de peso de los huevos desde el inicio de la incubación a la $1^{a}$ ovoscopía, fue en promedio de 0,11178 Kg (máximo 0,211 Kg y mínimo 0,086 Kg). La segunda ovoscopía se realizó a los 28 días. El peso promedio de los huevos fue $1,349 \mathrm{Kg}$. Luego de esta $2^{a}$ ovoscopía se descartaron 16 huevos, quedando 16 para que continúen el proceso de incubación. La pérdida de peso de los huevos desde la $1^{\mathrm{a}}$ a la $2^{\mathrm{a}}$ ovoscopía, fue en promedio $0,124 \mathrm{Kg}$ (máximo 0,216 Kg y mínimo: $0,089 \mathrm{Kg}$ ). Existe un nivel de correlación significativa entre los valores de pérdida de peso de los huevos desde el inicio de la incubación hasta la $1^{\mathrm{a}}$ ovoscopía, y la $1^{\mathrm{a}} \mathrm{y}$ $2^{\mathrm{a}}$ ovoscopía, lo que nos indica que existe una pérdida uniforme de peso en los huevos, acorde al desarrollo embrionario, sin cambios bruscos en la temperatura y humedad en el proceso de incubación.

Se obtuvo un $50 \%$ de fertilidad, que resulta un valor considerado regular para esta especie. Hay que considerar las condiciones peculiares con las que se ha trabajado a la fecha y que ya hemos puntualizado anteriormente. Los resultados que hemos logrado nos servirán para seleccionar adecuadamente los machos y las hembras para posteriores procesos. No se ha logrado obtener el nacimiento de ningún polluelo (\% incubabilidad 0), encontrándose a los 42- 43 días de incubación, y luego de la apertura forzada de los huevos, 12 polluelos con plumaje muertos con saco vitelino externo.

$$
\begin{gathered}
\text { Fertilidad }=\frac{\mathrm{N}^{\circ} \text { de huevos fértiles }}{\mathrm{N}^{\circ} \text { de huevos introducidos en la incubadora }} \times 100=50 \% \\
\text { Incubabilidad }=\frac{\mathrm{N}^{\circ} \text { de pollos nacidos }}{\mathrm{N}^{\circ} \text { de huevos fértiles }} \times 100=0
\end{gathered}
$$

Luego de realizados los aislamientos e identificación respectiva, se reconoció que en las muestras hay presencia de Escherichia coli y Bacillus $s p$, tanto de manera aislada como combinada, en todos los casos (muestras cloacales de las 9 hembras).

\section{DISCUSIÓN}

Las hembras No 1489 y 1405 las mejores ponedoras con 19 y 14 huevos respectivamente. Estas hembras probablemente estén dentro del rango esperado de postura si consideraríamos toda la estación reproductiva (unos 4-5 meses). El estado nutricional resulta fundamental para poder explicar esta deficiencia relativa en la postura de la mayoría de las hembras, pues en la EEA El Mantaro se adolece de un régimen alimenticio balanceado, acorde con las necesidades de postura de estos animales. Además el estado de los potreros donde pastan estos animales puede ser considerado de condición regular a pobre.

El almacenamiento previo no se ha producido, o este fue mínimo (2 días), mientras que muy pocos huevos tuvieron un almacenamiento de 6 días. Lo recomendable es de una semana.

Con referencia al desarrollo de los polluelos antes de la eclosión, corresponde a un desarrollo de 6 a 6,5 semanas. La causa de las mortalidades iniciales, intermedias y la de los polluelos in ovo puede ser atribuida principalmente a la altura (3 $200 \mathrm{nmsm}$ ) donde se encuentra la sala de incubación; a esta altitud, la tensión de oxígeno es muy baja y esto impide 0 dificulta el desarrollo del embrión. Esto mismo ha sido reportado ampliamente para procesos de incubación de huevos de gallina, con pobres resultados de incubabilidad y de eclosión. Según Zhang y col. (2008), el déficit de 02 es el factor principal que produce incubabilidad baja y calidad del polluelo pobre. Estos resultados son coherentes con los reportados por Stephens y Ploog (1967) en un ensayo realizado en el IVITA El Mantaro, Perú, a 3200 msnm. Un criterio importante es el de Deeming (2001), sobre la ventilación ya que el huevo necesitaría 5 veces más de oxígeno en la segunda mitad de la incubación. Según trabajos previos de Reiner (1995) el mayor consumo de oxígeno se daría el día 36 de incubación, con necesidades de unos 240 litros de aire fresco por huevo y día. Deeming recomienda bajar la temperatura en la fase final ya que los huevos grandes pueden tener problemas de eliminación del calor metabólico, constituyendo esto una causa añadida de mortalidad embrionaria.

El período más crítico es cuando se produce el cambio en la respiración del embrión, que pasa de ser corioalantoidea a pulmonar, es el momento en que se produce el $50 \%$ de las muertes independientemente si los resultados hubieran sido malos o exitosos. El 
período en el cual el embrión cesa de respirar a través de la membrana para comenzar a hacerlo por medio de sus pulmones dura cerca de 6 horas, de no ocurrir se produce la muerte embrionaria. Es importante apuntar que según las investigaciones de Deeming (1995), el mayor índice de mortalidad se produce al inicio de la incubación y al final de la misma.

Los microorganismos encontrados corresponden mayormente a los componentes de la flora microbiana normal de la cloaca, y que no podría sospecharse que sean las causales de contaminación del huevo que ponga en riesgo el desarrollo embrionario durante el proceso de incubación, aunque este hecho debería de corroborarse con nuevos estudios a nivel de los mismos huevos, luego de la postura, almacenaje y al inicio de la incubación, así como en los huevos descartados en el proceso de incubación.

\section{CONCLUSIONES}

- El número de huevos producidos para incubación fue de 64 en total con un promedio de 7,11 huevos por hembra para los dos meses de ciclo reproductivo, siendo las hembras № 1489 y 1405 las mejores ponedoras con 19 y 14 huevos respectivamente.

- El promedio del peso de los huevos fue $1,512 \mathrm{Kg}$, con un valor máximo de $1,687 \mathrm{Kg}$ y un valor mínimo de $1,285 \mathrm{Kg}$ (DS: 0,105 y Var: 0,011)
- Se realizaron 13 cargas de huevos a la incubadora, en promedio 4,92 huevos/carga, (rango 1 - 12). El peso promedio de los huevos/cargada fue 1,519, con un rango de 1,403 a 1,610 Kg.

- Las hembras Nos 1489 y 1405 tuvieron el mejor comportamiento en lo referente a su fertilidad, pues a la primera y segunda ovoscopía presentaron el mayor número de huevos en proceso de incubación.

- Los machos Nos 1339, 1355, 1457 y 1483 aparentemente no han logrado fecundar a las hembras. Los mejores machos en este rubro fueron los $\mathrm{N}^{0} \mathrm{~s} 2042$, 1412 y 1331.

- El macho № 2042 ha fertilizado un mayor número de huevos que han llegado casi a término de la incubación, seguido del macho $\mathrm{N}^{0}$ 1412, infiriéndose que podrían ser considerados como los mejores machos desde el punto de vista de fertilidad.

El peso promedio de los huevos a la $1^{\text {a }}$ ovoscopía fue de $1,453 \mathrm{Kg}$., descartándose 32 huevos y quedando 32 para que continúen el proceso de incubación.

- La pérdida de peso de los huevos desde el inicio de la incubación a la $1^{a}$ ovoscopía, en promedio 